\title{
Foundation of Hydrodynamics for Systems with Strong Interactions
}

\author{
Cheuk-Yin Wong \\ Physics Division, Oak Ridge National Laboratory, Oak Ridge, TN 37830
}

\begin{abstract}
For a dense and strongly interacting system, such as a nucleus or a strongly-coupled quark-gluon plasma, the foundation of hydrodynamics can be better found in the quantum description of constituents moving in the strong mean fields generated by all other particles. Using the result that the Schrödinger equation and the Klein-Gordon equation can be written in hydrodynamical forms, we find that the probability currents of the many-body system in the mean-field description obey a hydrodynamical equation with stress tensors arising from many contributions: quantum effects, mean-field interactions, and thermal fluctuations. The influence of various contributions to the hydrodynamical motion is expected to vary with the temperature, as the quantum and mean-field stress tensors playing more important roles at low and moderate temperatures.
\end{abstract}

Keywords: Schrödinger equation, Klein-Gordon equation, hydrodynamics

PACS: $03.65 . P m$ 47.10.A-

\section{INTRODUCTION}

The foundation of hydrodynamics is usually presented within the kinetic theory, in which particles are described as a gas with weak interactions [1]. For a dense and strongly interacting system, such as a nucleus or a strongly-coupled quark-gluon plasma, the foundation can be better found in a quantum description of a system with constituents moving in the strong mean fields generated by all other particles [2, 3]. As the timedependent Schrödinger equation can be cast into a hydrodynamical form [4, 2], the evolution of the probability currents in the mean-field potential provides support for the validity of the liquid-drop model of a nucleus [3], as in Bohr and Wheeler [5], and the validity of the dynamical model of nuclear fission, as in Hill and Wheeler [6]. The deviation of classical hydrodynamics from the quantum description is embodied in the presence of the quantum stress tensor $p_{i j}^{(q)}$ [2, 3]. Quantum shell effects manifest themselves as nuclear shell effects superimposed on a smooth hydrodynamical liquiddrop background, and they lead to the intrinsic deformation in many nuclei [7].

In particle and nuclear collisions at high energies, a phenomenological relativistic hydrodynamical description of the process is a reasonable concept, as pioneered by Landau [8] and supported by experimental findings [9, 10, 11]. In the related search for the foundation of relativistic hydrodynamics using the Klein-Gordon equation, we encounter the difficulty that the naive probability density constructed from the wave function of the Klein-Gordon equation may be negative. The presence of a negative probability density may appear to preclude its description in relativistic hydrodynamics. However, as pointed out by Feshbach and Villars [12], the negative probability density arises because the Klein-Gordon equation describes both particle and antiparticle degrees of freedom. 
Positive probability densities can be formulated when we separate out the particle and antiparticle degrees of freedom to write the Klein-Gordon equation as a set of two coupled time-dependent Schrödinger equations. We shall follow and modify the formalism of Feshbach and Villars [12] in order to search for the foundation for relativistic hydrodynamics in strongly-coupled systems.

\section{NON-RELATIVISTIC HYDRODYNAMICAL DESCRIPTION OF A NUCLEUS}

We begin by briefly summarizing the non-relativistic hydrodynamical description of a nucleus with strongly interacting constituents. As the Schrödinger equation for a singleparticle in an external field can be cast into the form of a hydrodynamical equation [2], a system of non-relativistic single particles interacting in their own mean fields can be described by non-relativistic hydrodynamics [2, 3]. We can represent the single-particle state wave function for a state $a$ in terms of the amplitude function $\phi_{a}$ and the phase function $S_{a}$ as

$$
\psi_{a}(\mathbf{r}, t)=\phi_{a}(\mathbf{r}, t) \exp \left\{-i S_{a}(\mathbf{r}, t)\right\}
$$

In the time-dependent mean-field description we obtain the Euler equation for the probability current $n u^{i}[3]$,

$$
\frac{\partial n u^{i}}{\partial t}+\sum_{j=1}^{3} n u^{i} u^{j}=-\frac{1}{m} \sum_{j=1}^{3} \frac{\partial}{\partial x_{j}}\left(p_{i j}^{(q)}+p_{i j}^{(t)}+p_{i j}^{(v)}\right),
$$

where $n=\sum_{a} n_{a} \phi_{a}^{2}, \quad u^{i}=\sum_{a} n_{a} \phi^{2} \nabla_{i} S_{a} / \sum_{a} n_{a} \phi_{a}^{2}$ for $i=1,2,3, n_{a}$ is the occupation number for the state $a$,

$$
\begin{gathered}
p_{i j}^{(q)}=-\frac{\hbar^{2}}{4 m} \sum_{a} n_{a} \nabla^{2} \phi_{a}^{2} \delta_{i j}+\frac{\hbar^{2}}{m} \sum_{a} n_{a} \nabla_{i} \phi_{a} \nabla_{j} \phi_{a}, \\
p_{i j}^{(t)}=\frac{\hbar^{2}}{m} \sum_{a} n_{a} \phi_{a}^{2}\left(\nabla_{i} S_{a}-m u^{i}\right)\left(\nabla_{j} S_{a}-m u^{j}\right), \\
\frac{\partial}{\partial x^{j}} p_{i j}^{(v)}(\mathbf{r}, t)=n(\mathbf{r}, t) \frac{\partial}{\partial x^{j}} \int d^{3} \mathbf{r}_{2} n\left(\mathbf{r}_{2}, t\right) v\left(\mathbf{r}, \mathbf{r}_{2}\right),
\end{gathered}
$$

and $v\left(\mathbf{r}, \mathbf{r}_{2}\right)$ is the two-body interaction that generates the mean field. The stress tensor due to the mean-field interaction can also be given as

$$
\frac{\partial}{\partial x^{j}} p_{i j}^{(v)}(\mathbf{r}, t)=n(\mathbf{r}, t) \frac{\partial}{\partial x^{j}}\left(\frac{\partial\left(W^{(v)} n\right)}{\partial n}\right),
$$


where $W^{(v)}$ is the energy per particle arising from the mean-field interaction. The meanfield stress tensor $p_{i j}^{(v)}$ is given explicitly by

$$
p_{i j}^{(v)}=\left\{n \frac{\partial\left(W^{(v)} n\right)}{\partial n}-W^{(v)} n\right\} \delta_{i j} .
$$

Thus, the total pressure arises from many sources: (i) quantum stress tensor $p_{i j}^{(q)}$ from quantum effects, (ii) the thermal stress tensor $p_{i j}^{(t)}$ from the deviation of the individual velocity fields from the local mean velocities, and the (iii) mean-field stress tensor $p_{i j}^{(v)}$ from the mutual interaction between fluid elements. For example, for a nucleus in which the nucleons interact with the Skyrm interaction, we have [3]

$$
p_{i j}^{(q)}=\frac{\hbar^{2}}{5 m}\left(\frac{6 \pi^{2}}{4}\right)^{2 / 3} n^{5 / 3} \delta_{i j}
$$

and

$$
p_{i j}^{(v)}=\frac{3}{8}\left(t_{0}+\frac{1}{3} t_{3} n\right) n^{2} \delta_{i j}
$$

where for the Skyrm I nucleon-nucleon interaction, $t_{0}=-1057 \mathrm{MeV} / \mathrm{fm}^{3}$ is the twobody interaction strength, and $t_{0}=+14463 \mathrm{MeV} / \mathrm{fm}^{6}$ is the three-body interaction strength.

A nucleus is a strongly-coupled system. The quantum and mean-field stress tensors are the dominant component for the nuclear fluid at low and moderate temperatures. The thermal stress tensor $p_{i j}^{(t)}$ can take on different values, depending on the occupation numbers of the single-particle states that determines the degree of thermal equilibrium of the system. For a thermally equilibrated system, the thermal stress tensor is

$$
p_{i j}^{(t)}=\frac{\hbar^{2}}{5 m}\left(\frac{6 \pi^{2}}{4}\right)^{2 / 3} n^{5 / 3}\left[\frac{2 m k T}{\hbar^{2} m\left(6 \pi^{2} n / 4\right)^{2 / 3}}\right]^{2} \delta_{i j},
$$

which is small for low and moderate temperatures. We observe that when $\left|p_{i j}^{(q)}+p_{i j}^{(v)}\right| \gg$ $p_{i j}^{(t)}$ for a strongly interacting system at low and moderate temperatures, there can be situations in which the system can behave quasi-hydrodynamically even though the state of the system has not yet reach thermal equilibrium, as is evidenced by the presence of nuclear collective vibrational and rotational states at low and moderate temperatures. In this case, the hydrodynamical state is maintained essentially by the quantum stress tensor and the strong mean-field stress tensor, and not by the thermal stress tensor. 


\section{RELATIVISTIC HYDRODYNAMICS AND THE KELIN-GORDON EQUATION}

In the collision of high-energy particles and nuclei, the relativistic hydrodynamics description was pioneered by Landau [8]. Subsequent considerations for the evolution of quark-gluon matter in the work of Bjorken [13], Baym et al. [14], Ollitraut [15, 16], and many others [17] lead to successful investigations of the hydrodynamics of strongly interacting matter in extreme conditions in relativistic heavy-ion collisions [9, 10, 11, 17].

In the search for the foundation of relativistic hydrodynamics using the Klein-Gordon equation, we can overcome the apparent difficulty of a possible negative naive probability density by re-writing the Klein-Gordon equation as a set of coupled time-dependent Schrödinger equations for particles and antiparticles, as in Feshbach and Villars [12].

A general solution of the Klein-Gordon equation contains two components that can be represented by a column vector,

$$
\Psi=\left(\begin{array}{l}
\chi_{+} \\
\chi_{-}
\end{array}\right),
$$

where $\chi_{+}$and $\chi_{-}$have positive norms, $\left|\chi_{ \pm}\right|^{2}$, which can be interpreted as the probability densities of particles and antiparticles respectively. We find it necessary, however, to modify the formulation of Feshbach and Villars [12] so as to obtain a set of coupled equations that facilitate the re-writing of the Klein-Gordon equation in the hydrodynamical form. We re-write the Klein-Gordon equation for the wave functions $\chi_{+}$and $\chi_{-}$as [18]

$$
\begin{aligned}
\left(i \hbar \partial_{t}-e_{ \pm} A^{0}\right) \chi_{ \pm} & =\frac{1}{2\left(E-e_{a} A^{0}\right)}\left\{\left(\frac{\hbar}{i} \nabla-e_{ \pm} \vec{A}\right)^{2}+(m+S)^{2}+\left[\left(E-e_{a} A^{0}\right)^{2}-i \hbar \partial_{t}\left(E-e_{a} A^{0}\right)\right]\right\} \chi_{ \pm} \\
& +\frac{1}{2\left(E-e_{a} A^{0}\right)}\left\{\left(\frac{\hbar}{i} \nabla-e_{ \pm} \vec{A}\right)^{2}+(m+S)^{2}-\left[\left(E-e_{a} A^{0}\right)^{2}-i \hbar \partial_{t}\left(E-e_{a} A^{0}\right)\right]\right\} \chi_{\mp}^{*},
\end{aligned}
$$

where $E$ is the positive expectation value of the operator $i \hbar \partial_{t}$ for the single-particle state, $e_{ \pm}= \pm e, e_{a}=v_{a} e$, and $v_{a}$ is the conserved particle number of the state.

\section{KLEIN-GORDON EQUATION IN HYDRODYNAMICAL FORM}

To obtain the equation of continuity and the Euler equation from Eq. (12), we write the wave functions $\chi_{ \pm}$in terms of the amplitude and phase functions as in Eq. (1),

$$
\chi_{ \pm}(\mathbf{r}, t)=\phi_{ \pm}(\mathbf{r}, t) \exp \left\{i S_{ \pm}(\mathbf{r}, t)\right\} .
$$

We construct $\chi_{ \pm}^{*} \times(12)-\chi_{ \pm} \times(12) *$ and obtain

$$
\partial_{t}\left[\left(E-e_{a} A^{0}\right) \phi_{ \pm}^{2}\right]+\nabla \cdot\left[\phi_{ \pm}^{2}\left(\nabla S_{ \pm}-e_{ \pm} \vec{A}\right)\right]=X_{ \pm},
$$

where 


$$
\begin{aligned}
2 X_{ \pm}= & \left\{\chi_{ \pm}^{*}\left(\frac{\hbar}{i} \nabla-e_{ \pm} \vec{A}\right)^{2} \chi_{\mp}^{*}-\chi_{ \pm}\left(\frac{\hbar}{-i} \nabla-e_{ \pm} \vec{A}\right)^{2} \chi_{\mp}\right\} \\
& +\left[(m+\mathscr{S})^{2}+\left(E-e_{a} A\right)^{2}\right]\left(\chi_{ \pm}^{*} \chi_{\mp}^{*}-\chi_{ \pm} \chi_{\mp}\right) \\
& +\left[i \hbar \partial_{t}\left(E-e_{a} A^{0}\right)\right]\left(\chi_{ \pm}^{*} \chi_{\mp}^{*}+\chi_{ \pm} \chi_{\mp}\right) .
\end{aligned}
$$

As the quantities $X_{+}$and $X_{-}$are not separately a full divergence, the total number of particles and antiparticles are not separately conserved. However, the difference of the particle number and antiparticle number of the two components satisfies the equation

$\partial_{t}\left[\left(E-e_{a} A^{0}\right)\left(\phi_{+}^{2}-\phi_{-}^{2}\right)\right]+\nabla \cdot\left[\phi_{+}^{2}\left(\nabla S_{+}-e_{+} \vec{A}\right)\right]-\nabla \cdot\left[\phi_{-}^{2}\left(\nabla S_{-}-e_{-} \vec{A}\right)\right]=X_{+}-X_{-}$

It can be shown that the difference $\left(X_{+}-X_{-}\right)$is a complete divergence. Therefore, the quantity

$$
v_{a}=\int d \mathbf{r} \frac{E-e_{a} A^{0}}{m}\left(\phi_{+}^{2}-\phi_{-}^{2}\right)=\int d \mathbf{r} \frac{E-e_{a} A^{0}}{m}\left(\left|\chi_{+}\right|^{2}-\left|\chi_{-}\right|^{2}\right)
$$

is a conserved quantity.

Hydrodynamical description will be appropriate after the active pair production stage has passed and the expansion of the system is driven by a slowly varying mean field. It is this type of motion, with the suppression of pair production, for which we wish to provide a hydrodynamical description. Under such a circumstance, one can speak of a single-particle system with a definite particle number $v_{a}$ which can take on the value $v_{a}=1$ for a particle and $v_{a}=-1$ for an antiparticle. From Eq. (12), the equation for the phase function $S_{ \pm}$for this simplified case is

$$
\begin{aligned}
\left(\partial_{t} S_{ \pm}+e_{ \pm} A^{0}\right)= & \frac{1}{2\left(E-e_{a} A^{0}\right)}\left\{\left[\left(\nabla^{2} \phi_{ \pm}\right) / \phi_{ \pm}-\left(\nabla S_{ \pm}-e_{ \pm} \vec{A}\right)^{2}\right]\right. \\
& \left.-(m+\mathscr{S})^{2}-\left(E-e_{a} A^{0}\right)^{2}\right\}
\end{aligned}
$$

For this case with suppressed pair production, $e_{a}=v_{a} e=e_{ \pm}$. We take the gradient $\nabla_{i}$ of the above for $i=1,2,3$, and multiply by $\phi_{ \pm}^{2}\left(E-e_{ \pm} A^{0}\right)$. Using the equation of continuity for this simplifying case with the suppression of pair production, we obtain

$$
\begin{aligned}
& \partial_{t}\left[\frac{\left(E-e_{ \pm} A^{0}\right) \phi_{ \pm}^{2}\left(\nabla_{i} S_{ \pm}-e_{ \pm} A^{i}\right)}{m+\mathscr{S}}\right]+\sum_{j=1}^{3} \nabla_{j}\left[\frac{\phi_{ \pm}^{2}\left(\nabla_{j} S_{ \pm}-e_{ \pm} A^{j}\right)\left(\nabla_{i} S_{ \pm}-e_{ \pm} A^{i}\right)}{m+\mathscr{S}}\right] \\
= & -\frac{m}{m+\mathscr{S}} \sum_{j=1}^{3} \nabla_{j} p_{i j}^{(q)}-\phi_{ \pm}^{2} \nabla_{i} \mathscr{S} \\
& +\frac{1}{m+\mathscr{S}}\left\{-\left(E-e_{ \pm} A^{0}\right) \phi_{ \pm}^{2} e_{ \pm} F^{0 i}-\sum_{j=1}^{3} \phi_{ \pm}^{2}\left(\nabla_{j} S_{ \pm}-e_{ \pm} A^{j}\right) e_{ \pm} F^{i j}\right\} \\
& \left.+\frac{e_{ \pm} \nabla_{i} A^{0} \phi_{ \pm}^{2}}{2\left(E-e_{ \pm} A^{0}\right)(m+\mathscr{S})}\left\{\left(\nabla^{2} \phi_{ \pm}\right) / \phi_{ \pm}-\left(\nabla S_{ \pm}-e_{ \pm} \vec{A}\right)^{2}\right]-(m+\mathscr{S})^{2}+\left(E-e_{ \pm} A^{0}\right)^{2}\right\}
\end{aligned}
$$




$$
\begin{aligned}
& -\left(E-e_{ \pm} A^{0}\right) \phi_{ \pm}^{2}\left(\nabla_{i} S_{ \pm}-e_{ \pm} A^{i}\right) \frac{\partial_{t} \mathscr{S}}{(m+\mathscr{S})^{2}} \\
& -\sum_{j=1}^{3}\left[\frac{\phi_{ \pm}^{2}\left(\nabla_{j} S_{ \pm}-e_{ \pm} A^{j}\right)\left(\nabla_{i} S_{ \pm}-e_{ \pm} A^{i}\right)}{m+\mathscr{S}}\right] \frac{\nabla_{j} \mathscr{S}}{(m+\mathscr{S})^{2}}
\end{aligned}
$$

We can identity the fluid energy density $\varepsilon_{ \pm}$as

$$
\varepsilon_{ \pm}=(m+\mathscr{S}) \phi_{ \pm}^{2}
$$

as it corresponds to the fluid energy density for a fluid element at rest. The fluid is characterized by a relativistic 4 -velocity $u^{\mu}$. We can identify

$$
u_{ \pm}^{0}=\frac{E-e_{ \pm} A^{0}}{m+\mathscr{S}}, \text { and } u_{ \pm}^{i}=\frac{\nabla_{i} S_{ \pm}-e_{ \pm} A^{i}}{m+\mathscr{S}}, \quad \text { for } i=1,2,3
$$

which obeys $\left(u_{ \pm}^{0}\right)^{2}-\left(\vec{u}_{ \pm}\right)^{2}=1$ in the absence of the quantum effects. We can write the equation of motion for the probability currents in terms of the hydrodynamical equation

$$
\begin{aligned}
& \partial_{t}\left(\varepsilon_{ \pm} u_{ \pm}^{0} u_{ \pm}^{i}\right)+\sum_{j=1}^{3} \nabla_{j} \varepsilon_{ \pm} u_{ \pm}^{i} u_{ \pm}^{j}+\frac{m}{m+\mathscr{S}} \sum_{j=1}^{3} \nabla_{j} p_{ \pm i j}^{(q)} \\
= & -\phi_{ \pm}^{2} \nabla_{i} \mathscr{S}+\frac{1}{m+\mathscr{S}}\left\{-\left(E-e_{ \pm} A^{0}\right) \phi_{ \pm}^{2} e_{ \pm} F^{0 i}-\sum_{j=1}^{3} \phi_{ \pm}^{2}\left(\nabla_{j} S_{ \pm}-e_{ \pm} A^{j}\right) e_{ \pm} F^{i j}\right\} \\
& \left.+\frac{e_{ \pm} \nabla_{i} A^{0} \phi_{ \pm}^{2}}{2\left(E-e_{ \pm} A^{0}\right)(m+\mathscr{S})}\left\{\left(\nabla^{2} \phi_{ \pm}\right) / \phi_{ \pm}-\left(\nabla S_{ \pm}-e_{ \pm} \vec{A}\right)^{2}\right]-(m+\mathscr{S})^{2}+\left(E-e_{ \pm} A^{0}\right)^{2}\right\} \\
& -\left(E-e_{ \pm} A^{0}\right) \phi_{ \pm}^{2}\left(\nabla_{i} S_{ \pm}-e_{ \pm} A^{i}\right) \frac{\partial_{t} \mathscr{S}}{(m+\mathscr{S})^{2}} \\
& -\sum_{j=1}^{3}\left[\frac{\phi_{ \pm}^{2}\left(\nabla_{j} S_{ \pm}-e_{ \pm} A^{j}\right)\left(\nabla_{i} S_{ \pm}-e_{ \pm} A^{i}\right)}{m+\mathscr{S}}\right] \frac{\nabla_{j} \mathscr{S}}{(m+\mathscr{S})^{2}},
\end{aligned}
$$

where $i=1,2,3$. This is the Klein-Gordon equation in hydrodynamical form for its particle and antiparticle probability currents in external fields for the case with the suppression of pair production. The first two terms on the left-hand side correspond to $\partial_{\mu} T_{ \pm}^{\mu i}$ with $T_{ \pm}^{\mu i}=\varepsilon_{ \pm} u_{ \pm}^{\mu} u_{ \pm}^{i}$ for $\mu=0,1,2,3$. The third term on the left-hand side is the quantum stress tensor arising from the spatial variation of the amplitude of the single-particle wave function [2],

$$
p_{ \pm i j}^{(q)}=-\frac{\hbar^{2}}{4 m} \nabla^{2} \phi_{ \pm}^{2} \delta_{i j}+\frac{\hbar^{2}}{m} \nabla_{i} \phi_{ \pm} \nabla_{j} \phi_{ \pm}
$$

which comes from the quantum nature of the fluid. The first two terms on the righthand side contain forces coming from the scalar interaction, the electric field $F^{0 i}$ and the magnetic field $F^{i j}$, as in classical considerations. The third term on the right-hand side is the relativistic correction to the time-like part of the vector interaction, and the 
last two terms represent relativistic corrections associated with the spatial and temporal variation of the scalar interaction. Thus the dynamics of the probability currents obey a hydrodynamical equation, with forces on the fluid element arising from what one expects in physical and classical considerations. The additional element is the presence of the quantum stress tensor $p_{i j}^{(q)}$ that comes from the quantum nature of the fluid.

\section{RELATIVISTIC HYDRODYNAMICS FOR A MANY-BODY SYSTEM IN THE MEAN-FIELD DESCRIPTION}

A many-body system in the time-dependent mean-field description consists of a collection of independent particles moving in a time-dependent self-consistent mean-field potential generated by all other particles [2, 3, 19]. Each single-particle state $\psi_{a}$ is characterized by a state label $a$, particle number $v$, energy $E_{a}$, and occupation number $n_{a}$. For simplicity, we consider the case in which the mean-field potential arises from a scalar two-body interaction $v_{s}\left(\mathbf{r}_{1}, \mathbf{r}_{2}\right)$ and a time-like vector interaction $v_{0}\left(\mathbf{r}_{1}, \mathbf{r}_{2}\right)$. We further neglect the last three terms on the right-hand side of Eq. (22) which represent higher-order relativistic corrections. The equation of motion for the energy density $\varepsilon_{a v}$ and velocity fields $u_{a v}^{i}$ for $i=1,2,3$ in the single particle state $a$ and particle type $v$ is then

$$
\begin{aligned}
& \partial_{t}\left(\varepsilon_{a v} u_{a v}^{0} u_{a v}^{i}\right)+\sum_{j=1}^{3} \nabla_{j} \varepsilon_{a v} u_{a v}^{i} u_{a v}^{j}+\frac{m}{m+\mathscr{S}} \sum_{j=1}^{3} \nabla_{j} p_{(a v) i j}^{(q)} \\
= & -\phi_{a v}^{2} \nabla_{i} \mathscr{S}+\frac{E-e_{a v} A_{ \pm}^{0}}{m+\mathscr{S}} \phi_{a v}^{2} e_{a v} \frac{\partial A^{0}}{\partial x^{i}}
\end{aligned}
$$

where, in the frame with fluid element at rest,

$$
\mathscr{S}(\mathbf{r}, t)=\int d^{3} \mathbf{r}_{2} n\left(\mathbf{r}_{2}, t\right) v_{s}\left(\mathbf{r}, \mathbf{r}_{2}\right)
$$

and

$$
A^{0}(\mathbf{r}, t)=\int d^{3} \mathbf{r}\left\{n_{+}\left(\mathbf{r}_{2}, t\right) e_{+}+n_{-}\left(\mathbf{r}_{2}, t\right) e_{-}\right\} v_{0}\left(\mathbf{r}, \mathbf{r}_{2}\right)
$$

Here $n_{+}=\sum_{+} n_{a+} \phi_{a+}^{2}, n_{-}=\sum_{-} n_{a-} \phi_{a-}^{2}$, and $n=n_{+}+n_{-}$. We consider a strongly interacting system in which the number of particles and antiparticles are equal so that $n_{+}\left(\mathbf{r}_{2}\right)=n_{-}\left(\mathbf{r}_{2}\right)$ and $n_{+}\left(\mathbf{r}_{2}\right) e_{+}+n_{-}\left(\mathbf{r}_{2}\right) e_{-}$is zero. Then the contribution from the second term on the right-hand side of Eq. (24) is zero. Multiply Eq. (24) by $n_{a v}$ and sum over $\{a, v\}$, we get

$$
\begin{aligned}
& \partial_{t}\left(\sum_{a v} n_{a v} \varepsilon_{a v} u_{a v}^{0} u_{a v}^{i}\right)+\sum_{j=1}^{3} \nabla_{j}\left(\sum_{a v} n_{a v} \varepsilon_{a v} u_{a v}^{i} u_{a v}^{j}\right) \\
& +\frac{m}{m+\mathscr{S}} \sum_{j=1}^{3} \nabla_{j}\left(\sum_{a v} n_{a v} p_{(a v) i j}^{(q)}\right)+\left(\sum_{a v} n_{a v} \phi_{a v}^{2}\right) \nabla_{i} \mathscr{S}=0 .
\end{aligned}
$$


We define the total energy density $\varepsilon$ by

$$
\sum_{a v} n_{a v} \varepsilon_{a v}=\varepsilon
$$

and the average 4-velocity $u$ by

$$
u=\sum_{a v} n_{a v} \varepsilon_{a v} u_{a v} / \varepsilon
$$

We can introduce the thermal stress tensor $p_{i j}^{(t)}$ for $\{i, j\}=1,2,3$ as the correlation of the deviations of the single-particle velocity fields from the average

$$
\sum_{a v} n_{a v} \varepsilon_{a v}\left(u_{a v}^{i}-u^{i}\right)\left(u_{a v}^{j}-u^{j}\right) \equiv p_{i j}^{(t)}
$$

For the case with the suppression of pair production, we obtained the equation of hydrodynamics

$$
\partial_{t}\left(\varepsilon u^{0} u^{i}\right)+\sum_{j=1}^{3}\left\{\nabla_{j}\left(\varepsilon u^{i} u^{j}+p_{i j}^{(t)}+p_{i j}^{(v)}\right)+\frac{m}{m+\mathscr{S}} \nabla_{j} p_{i j}^{(q)}\right\}=0,
$$

where the total quantum stress tensor is

$$
p_{i j}^{(q)}=-\frac{\hbar^{2}}{4 m} \nabla^{2} \sum_{a v} n_{a v} \phi_{a v}^{2} \delta_{i j}+\frac{\hbar^{2}}{m} \sum_{a v} n_{a v} \nabla_{i} \phi_{a v} \nabla_{j} \phi_{a v}
$$

and the pressure due to the interaction $p_{i j}^{(v)}$ is

$$
\frac{\partial}{\partial x^{j}} p_{i j}^{(v)}(\mathbf{r}, t)=n(\mathbf{r}, t) \nabla_{i} \mathscr{S}(\mathbf{r}, t)=n(\mathbf{r}, t) \frac{\partial}{\partial x^{j}} \int d^{3} \mathbf{r}_{2} n\left(\mathbf{r}_{2}, t\right) v_{s}\left(\mathbf{r}, \mathbf{r}_{2}\right)
$$

The mean-field stress tensor $p_{i j}^{(v)}$ can also be given as

$$
p_{i j}^{(v)}=\left\{n \frac{\partial\left(W^{(v)} n\right)}{\partial n}-W^{(v)} n\right\} \delta_{i j}
$$

where $W^{(v)}$ is the energy per particle arising from the mean-field interaction. As an illustrative example, we can consider a density-dependent two-body interaction

$$
v_{S}\left(\mathbf{r}, \mathbf{r}_{2}\right)=\left[a_{2}+a_{3} n\left(\left(\mathbf{r}+\mathbf{r}_{2}\right) / 2\right)\right] \delta\left(\mathbf{r}-\mathbf{r}_{2}\right) .
$$

The contribution of the mean-field interaction to the stress tensor is then

$$
p_{i j}^{(v)}=\frac{1}{2}\left[a_{2}+2 a_{3} n(\mathbf{r})\right] n^{2} \delta_{i j}
$$


whose magnitude increases with the density and the strengths of the interaction.

The thermal stress tensor $p_{i j}^{(t)}$ can take on different values, depending on the occupation numbers of the single-particle states that determine the degree of thermal equilibrium of the system. In the time-dependent mean-field description, the motion of each particle state can be individually followed [19]. The occupation numbers $n_{a v}$ of the single-particle states will remain unchanged, if there are no additional interaction between the single particles due to the residual interactions. When particle residual interactions are allowed as in the extended time-dependent mean-field approximation [20], the occupation numbers will change and will approach an equilibrium distribution as time proceeds.

We note that as in the non-relativistic case, the total pressure arises from many sources. We come again to the observation that in situations when $\left|p_{i j}^{(q)}+p_{i j}^{(v)}\right| \gg$ $p_{i j}^{(t)}$ for a strongly-coupled system at low and moderate temperatures, there can be situations when the system behaves quasi-hydrodynamically, even though the state of the system has not yet reached thermal equilibrium. In this case, the hydrodynamical state is maintained mainly by the quantum stress tensor and the strong mean fields.

\section{SUMMARY AND DISCUSSIONS}

For dense systems with strongly interacting constituents, a reasonable description of the system can be formulated in terms of constituents moving in the strong mean fields generated by all other particles. From such an analysis, we find that the probability currents of the system obey a hydrodynamical equation with the stress tensor arising from many contributions. There is the quantum stress tensor that arises from quantum effects, there is the thermal stress tensor that arises from the deviation of the singleparticle velocity fields from the average velocity fields, and there is the mean-field stress tensor that arises from the mean-field interactions.

The importance of the three different contributions depend on the physical situations that are present in the system. For low temperature dynamics for which the quantum effects and mean fields are important, the dynamics of the strongly-coupled system will be dominated by the quantum stress tensor and the mean-field stress tensor. In that case, the degree of thermalization is of less significance as the thermal stress tensor plays a minor role. On the other hand, for very high temperatures for which the magnitude of the temperature far exceeds the strengths of the mean-field interactions and the quantum pressure, the thermal stress tensor plays the dominant role. The magnitude of the thermal stress tensor will depend sensitively on the degree of thermalization of the system. In between these limits, one can envisage the transition from the quantum and mean field dominating strongly-coupled regime to the thermal pressure dominating weakly-coupled regime as temperature increases.

What we have discussed is only a theoretical framework that exhibits clearly the different sources of stress tensors. To study specifically the dynamics of the quark-gluon plasma for example, it will be necessary to investigation the specific nature of different constituents and their interactions. Nevertheless, the general roles played by the different components of stress tensors can still be a useful reminder on the importance of the 
quantum and mean-field stress tensors in the strongly-coupled regime, at temperature just above the transition temperature $T_{c}$. The dominance of the quantum and meanfield stress tensors over the thermal stress tensor may imply that hydrodynamics may commence at an early stage even when thermal equilibrium has not been reached, as the total stress tensor may be hardly affected by the variation of the thermal stress tensor. It will therefore be of great interest to study whether the fast onset of the hydrodynamical motion as suggested by the elliptic flow measurements [16] may be caused by the dominance of the mean-field and quantum stress tensors for a strongly-coupled quarkgluon plasma. For $T$ slightly greater than $T_{c}$ such a possibility may perhaps be the case.

\section{ACKNOWLEDGMENTS}

The author wishes to thank Prof. H. W. Crater and for helpful discussions.

\section{REFERENCES}

1. See for example, K. Huang, Statistical Mechanics, J. Wiley \& Sons, N.Y. 1963; L.S. Garcia-Colin and A. Sandoval-Villalbazo, J.Nonequil.Thermo. 31, 11 (2006).

2. C. Y. Wong, J. Math. Phys. 17, 1008 (1976).

3. C. Y. Wong, J. A. Maruhn, and T. A. Welton, Nucl. Phys. A253, 469 (1975); C. Y. Wong, T. A. Welton, and J. A. Maruhn, Phys. Rev. C15, 1558 (1977); C. Y. Wong and J. A. McDonald, Phys. Rev. C16, 1196 (1977), C. Y. Wong, Phys. Rev. C17, 1832 (1978), C. Y. Wong and H. H. K. Tang, Phys. Rev. C20, 1419 (1979), C. Y. Wong, Phys. Rev. C 25, 1460 (1982).

4. E. Madelung, Z. Phys. 40, 332 (1926).

5. N. Bohr and J. A. Wheeler, Phys. Rev. 56, 426 (1939).

6. D. L. Hill and J. A. Wheeler, Phys. Rev. 89, 1102 (1953).

7. M. Brack, J. Damgaard, A.S. Jensen, H. C. Pauli, V. M. Strutinsky, and C. Y. Wong, Rev. Mod. Phys. 44, 320 (1972).

8. L. D. Landau, Izv. Akad. Nauk SSSR 17, 51 (1953), S. Z. Belenkij and L. D. Landau, Usp. Fiz. Nauk 56, 309 (1955); Nuovo Cimento, Suppl. 3, 15 (1956).

9. M. Murray, for the BRAHMS Collaboration, J.Phys. G30 S667 (2004); M. Murray, for the BRAHMS Collaboration, J. Phys. G35, 044015 (2008).

10. P. Steinberg, Nucl. Phys. A752, 423 (2005).

11. C. Y. Wong, Phys. Rev. C78, 054902 (2008); C. Y. Wong, arXiv:0809.0517

12. H. Feshbach and F. Villars, Rev. Mod. Phys. 30, 24 (1958).

13. J. D. Bjorken, Phys. Rev. D27, 140 (1983).

14. G. Baym, B. L. Friman, J.-P. Blaizot, M. Soyeur, and W. Czyz, Nucl. Phys. A407, 541 (1983).

15. J. Y. Ollitrault, Phys. Rev. D 46, 229, (1992).

16. J. Y. Ollitrault, Eur. J. Phys. 29, 275 (2008).

17. L. P. Csernai, Introduction to Relativistic Heavy-Ion Collisions, Willey, 1994; D. H. Rischke and M. Gyulassy, Nucl. Phys. A608, 479 (1996); D. Teaney, Phys. Rev. C68, 034913 (2004); T. Hirano and Y. Nara, Nucl. Phys. A743, 395 (2004); P. F. Kolb and U. Heinz.nucl-th/0305084(2003); P. Huovinen and P. V. Ruuskanen, Ann. Rev. Nucl. Par. Sci. (2006); C. Nonaka and B. A. Bass, Phys. Rev. C75, 014902 (2007); O. J. Socolowski, F. Grassi, Y. Hama, and T. Kodama, Phys. Rev. Lett. 93, 182903 (2004); W. N. Zhang, M. J. Efaaf, and C. Y. Wong, Phys. Rev. C70, 024903 (2004); T. Csorgo, F. Grassi, Y. Hama, and T. Kodama, Phys. Lett. 565, 107 (2003); T. Csorgo et al., Phys. Lett. B663, 306 (2008); R. Peschanski and E. N. Saridakis, Phys. Rev. C80, 024907 (2009); C. Y. Wong, Introduction to High-Energy Heavy-Ion Collisions, World Scientific Publisher, 1994.

18. C. Y. Wong, arXiv:1011.5510 (2010), accepted for publication in Journal of Mathematical Physics.

19. P. Bonche, S. Koonin, and J. W. Negele, Phys. Rev. C 13, 1226 (1976) .

20. C. Y. Wong and H. H. K. Tang, Phys. Rev. Lett. 40, 1070 (1978) ; C. Y. Wong and H. H. K. Tang, Phys. Rev. C20, 1419 (1979) 